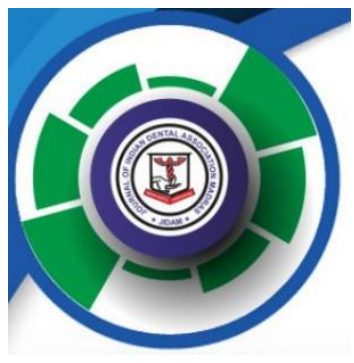

REVIEW ARTICLE

\title{
LIPOXINS - THE CRUCIAL FACTOR FOR RESOLUTION OF INFLAMMATION
}

\author{
Dr. Jaishree Tukaram Kshirsagar, Dr. NarmathaDevi. N, Dr. Sangeetha S, Dr. Priyangha. P. T
}

Department of Periodontics, Tamilnadu Government Dental College and Hospital, Chennai 600003, Tamilnadu, India

DOI:10.37841/jidam_2021_V8_I1_06

Address for Correspondence

Dr. N. NarmathaDevi, Post Graduate Student,

Department of Periodontics,

Tamilnadu Government Dental College and Hospital, Chennai 600003, Tamilnadu, India

Email id: narmathanatarajan1292@gmail.com

Received: 09.02.2021 First Published: 13.03.2021

Accepted: 10.03.2021

Published: 27.03.2021

\begin{abstract}
Eliminating offending pathogens from the site of infection is an effective host defense mechanism resulting in subsequent resolution of inflammation restoring tissue homeostasis. Lipoxins are endogenous proresolving mediators which also have anti-inflammatory properties. Lipoxins play a crucial role in suppressing exaggerated tissue injury and chronic inflammation. Several studies demonstrated that synthesized lipoxin anolog has the ability to inhibit neutrophil recruitment and alter vascular permeability. This review explains lipoxins generation, its actions at the site of inflammation, its role in periodontal disease and how it could be used as a potential drug.

KEY WORDS: Lipoxins, Defense mechanism, Inflammation, Resolution, Endogenous, Periodontal Disease
\end{abstract}




\section{INTRODUCTION}

Acute inflammation is a physiological innate immune response and its primary role is protective to host which is aimed to remove pathological noxious stimuli and to restore homeostasis ${ }^{1}$. The process of inflammation is often initiated by neutrophils in response to microbial challenge.

The fate of inflammation is determined by balance between the proinflammatory mediators and antiinflammatory mediators which neither amplifies the inflammation nor controls the inflammation. Thus, resolution is the ideal outcome of acute inflammation but its perpetuation leads to pathology. Resolution of inflammation is a highly ordered process regulated by numerous lipid mediators which are derived from essential fatty acids. These lipid mediators or resolution agonists are collectively called as specialised lipid mediators or immunoresolvents. Lipoxin is one of these specialised lipid mediators which is an acronym of lipoxygenase interaction products, first discovered class of immunoresolvents and is followed by lipoxin epimers called Aspirin triggered lipoxins. Evidence states that resolution is mediated by mediators like Arachidonic acid derived lipoxins, aspirin triggered Lipoxins, Resolvins E and Dseries, Maresins, Protectins. ${ }^{1}$

\section{LIPOXINS}

Lipoxins are the naturally occurring proresolving molecules produced from endogenous fatty acids by the action of lipoxygenase enzyme. It has potent antiinflammatory and resolution properties. Lipoxins also acts as inhibitors of neutrophils recruitment and stimulated macrophage recruitment.

\section{HISTORY}

Lipoxins were first described by Serhan, Hamberg, Nobel lacerate Samuelson in $1984^{1,2,3}$. These are initially reported in human blood neutrophils and when stimulated produces two lipoxins namely LXA4, LXB4. Later several studies have identified epimers of these two lipoxins, they are epilipoxins - 15epi-LXA4 and 15epi-LXB4.

\section{BIOCHEMISTRY}

Lipoxins are arachidonic acid derived metabolites which contains 3 hydroxyl residues and 4 double bonds and contains a trihydroxy tetraene group. This structural definition is distinguishing character from the other specialised lipid mediators such as resolvins and Maresins ${ }^{3}$.

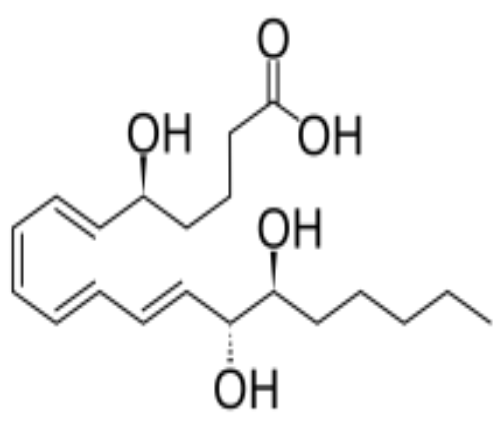

\section{SYNTHESIS}

Lipoxins are produced in the vessel lumen primarily through platelet and leukocyte transcellular biosynthesis ${ }^{1}$. Lipoxin are produced via several different pathways, by cell to cell interaction. ${ }^{2}$ These cell to cell interactions may also stimulate transcellular biosynthetic pathways and subsequent amplification signals like leukotrienes and prostaglandins or breaking signals involving novel compounds $^{2}$. Generation of lipoxins is a rapid process which is activated by inflammation, thrombosis and atherosclerosis (levy et al).

\section{I5-Lipoxygenase - initiated pathway}

Insertion of oxygen molecule into 15-carbon position (C15) of arachidonic acid is the essential step for lipoxin generation and this pathway requires the enzyme 15 lipoxygenase. Once the $\mathrm{C} 15$ position gets oxygenated; Arachidonic acid is converted into 15-hydroperoxy eicosatetraenoic acid (15-HPETE), which is a substrate for 5 -lipoxygenase present in the leukocytes. This was demonstrated by Serhan et al 1984, Ford Hut Chinsro $1991^{4}$.

This HPETE molecule is quickly converted into $15 \mathrm{~s}$ epoxytetraene by hydrolases. This forms the major route for Lipoxin generation.

\section{Lipoxygenase-Initiated Pathway}

The second route of lipoxin synthesis occurs by the interaction of neutrophils and platelets in the blood vessels. This cell to cell interaction involves 5-LO in neutrophils and 12-LO in platelets for the insertion of oxygen molecules into Arachidonic acid.

\section{Additional pathways for Lipoxin synthesis:}

Neutrophils synthesized LIA4 that is converted in epithelial cells by $15 \mathrm{LO}$, especially in tracheal epithelial cells can also produce lipoxins by LIA4 dependant mechanism. Another route often involves 5,6 dihydroxyeicosane which are the substrates for the 
Jaishree et al: Lipoxins for Resolution of Inflammation

conversion of lipoxin which include both 15-LO and 12LO enhanced by cell to cell adhesion interactions ${ }^{6}$.

\section{Aspirin triggered lipoxins:}

In transcellular biosynthesis with the presence of aspirin, COX-switches its catalytic activity and generates 15R-HETE instead of prostaglandins. Thereby Aspirin inhibits prostaglandin synthesis by both COX-1and COX2 pathways. When COX- acetylated by aspirin in epithelial or endothelial cells, arachidonic acid converts into 15RHETE, which is transformed via transcellular routes to produce 15-epilipoxins by leukocytes. The activated and adherent leukocytes releases its 5-LO and converts $15 \mathrm{R}$ HETE into 5-epoxytetraene within leukocytes. This conversion results in generation of 15-epi-LXA4 and 15epi-LXB4. 15epi-LXA4 is highly potent than native LXA4 in inhibiting the adhesion of neutrophils. This ATL can serve as a potent endogenous anti-inflammatory signals or mediators of some aspirin related beneficial actions like MI prevention.

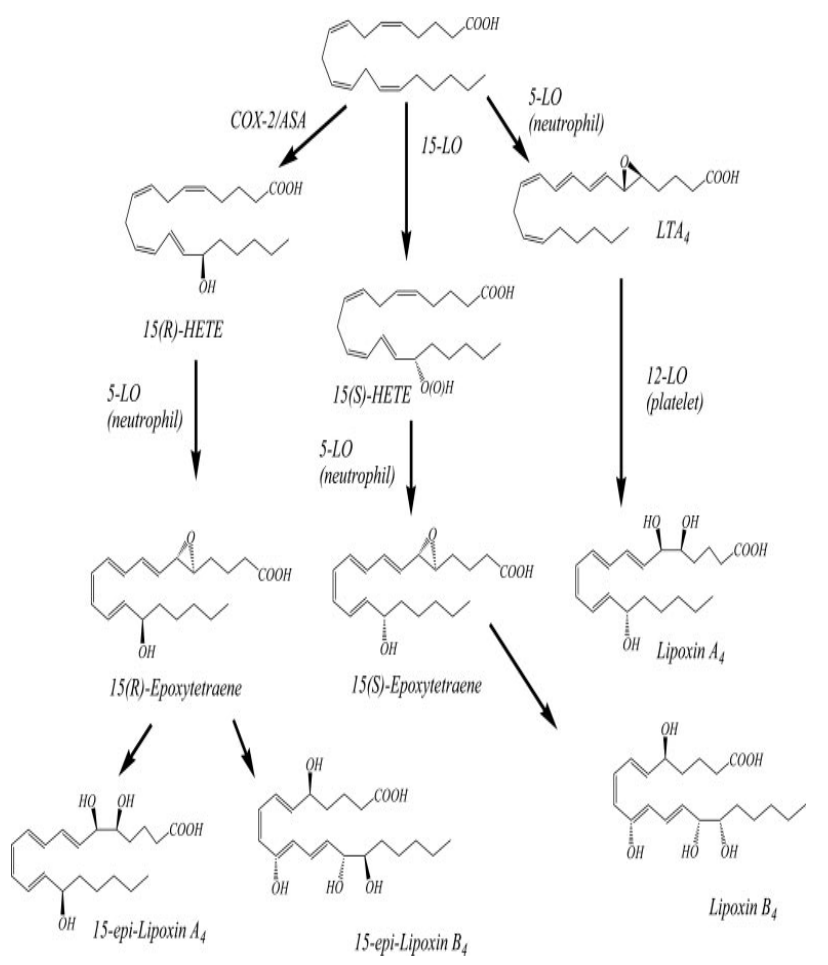

\section{LIPOXINS AT THE SITE OF INFLAMMATION}

Neutrophils play a critical role in the acute immune response against microbial challenge. Recruitment of neutrophils to the inflammation site and subsequent clearance helps to resolve inflammation. Lipoxins help in neutrophil migration to the inflammation site by increasing levels of cytosolic calcium which helps the neutrophils to put out their pseudopods and initiates the migration of neutrophils. Lipoxins also help in neutrophil clearance at the site of infection there by resolving the inflammation.
Myeloperoxidase rescues the PMNs from constitutive apoptosis thereby prolongs the lifespan of PMNs through adhesion molecule cluster differentiation CD11b/CD18. Lipoxins reduce IL- 8 and nitrous oxide which helps in prevention of tissue injury and attenuates the accumulation of nuclear factor activator protein-1 in the nucleus which further reduces IL-8 production thereby reduces neutrophils accumulation $\&$ activation ${ }^{9}$.

Lipoxins often promotes resolution of inflammation by delaying the apoptosis of macrophages and stimulates rapid activation of $\mathrm{P} 13 \mathrm{~K} / \mathrm{Akt} \& \mathrm{ECK} / \mathrm{Nrf} 2$ pathways which has a relevant role in macrophage apoptosis.

\section{LIPOXINS AND DISEASE:}

The lipoxins in clinical inflammation was initially demonstrated by Lee et al 1990 in bronchial lavage. The LXA4 and LXB4 have been formed in nasal polyps. The LXA4 is found in the nasal lavage obtained from aspirin sensitive asthmatics. Lipoxins serves as a useful biomarker of asthma and for long term clinical improvement in arthritis patients. During chronic myeloblastic leukemia, platelets lose 1-lipoxygenase and lose their ability to produce lipoxins which may be related to blast crisis (Stenke et al). A reduction and alteration in lipoxin synthesis has been found in chronic liver disease $\&$ in chronic myelogenous leukemia.

\section{LIPOXINS IN PERIODONTAL DISEASE}

Inducible cycloxygenase isoform involvement and the novel role of lipid mediators are studied and data derived from these studies have showed that COX-2 may have multiple role in development and progression of periodontal diseases which has been demonstrated by Pouliol et al 2000. Samples from crevicular fluid of localised aggressive periodontitis patients exhibits prostaglandins $\mathrm{E}_{2}, 5 \mathrm{LO}$ derived products, LTB4 and biosynthesis products like LXA4. The neutrophils have found to generate significant arachidonic acid metabolite. These findings suggest that neutrophils influences the pathogenesis of periodontal disease in several ways ${ }^{10}$.

The role of lipid mediators in the neutrophils response to P.gingivalis was studied in animal model. When P.gingivalis was injected into murine dorsal air pouches 1- leukocytes was stimulated, 2- levels of $\mathrm{PGE}_{2}$ increased. In addition upregulation of COX-2 mRNA expression was also demonstrated ${ }^{10}$.

Metabolically stable lipoxin analogs administration potentially blocked the neutrophils trafficking into dorsal pouch cavity and also reduced $\mathrm{PGE}_{2}$ levels within the exudates without allowing the spread of infections. These 
Jaishree et al: Lipoxins for Resolution of Inflammation

results suggest that neutrophils may provide an important source of $\mathrm{PGE}_{2}$ in periodontal tissues ${ }^{10}$. Moreover these data also provide strong evidence that lipoxins may have a protective role in periodontitis by limiting further recruitment of neutrophils, neutrophils mediated injuries and subsequent reduction of inflammatory responses that prevent tissue invasion by periodontal pathogens. It has been proposed that lipoxin generation and its relationship to $\mathrm{PGE}_{2}$ and $\mathrm{LTX}_{4}$ can be a significant marker in periodontal disease and also suggest that activated neutrophils from localised aggressive periodontitis patients produced it $^{10}$.

\section{LIPOXINS AS A POTENTIAL DRUG}

Several studies have demonstrated that lipoxins and synthetic analogues protects tissues from acute and chronic inflammation and the mechanism include downregulation of pro-inflammatory cytokines and chemokines ( e.g., interleukin- $1 \beta$ and tumor necrosis factor- $\alpha$ ), inhibition of activation of the master proinflammatory pathway(e.g nuclear factor $\kappa$-light chain - enhancer of activated B cell pathway) and increased release of the pro-resolving cytokines (e.g., interleukin - 10). Three generations of LXs analogues are well described in the literature, and more recently fourth generation has been generated that appears to show enhanced potency. In this review, we will briefly discuss the potential therapeutic opportunity provided by lipoxin $\mathrm{A}_{4}$ as a novel approach to treat chronic inflammatory disorders ${ }^{14}$.

Lipoxin stable analogs were analysed for their clinical inhibition of inflammation and are produced by means of recombinant dehydrogenous screen assay which is an inexpensive and rapidly to design. These analogs synthesized were tested for their ability to inhibit the neutrophil recruitment and alteration in vascular permeability in several murine models.

An experimental study was done by Serhan et al where they used rabbit models, animals were allocated into two groups, and both the groups were ligatured and were inoculated with $P$. gingivalis. One group received LX (6microgram in 6microL) three times a week applied topically and the other group received ethanol alone. At 6 weeks, animals were sacrificed and jaws were harvested for analysis of bone loss which was done using an X-ray. Statistically significant reduction in all disease parameters including bone loss was noticed in the group which was treated with LX. Van Dyke and Serhan concluded that application of LX in the management of periodontitis could inhibit more than $90 \%$ of bone loss. ${ }^{15}$

\section{CONCLUSION}

Lipoxin and ATL are the first recognized members of a new class of endogenous mediators which has antiinflammatory properties and serves for the preresolution of inflammation. $\mathrm{PGE}_{2}$ may appear to have antiinflammatory properties in certain conditions. But in most cases it enhances inflammation in-vivo. This is may be due to presence of numerous receptor isoforms and differential coupled mechanisms of $\mathrm{PGE}_{2}$ and its diversity in human physiology. Because the integrated response of the host is necessary to health and disease, it is essential to achieve a more complete understanding of the molecular and cellular events, their formation, the actions of endogenous mediators of inflammation to control the intensity and duration of inflammation.

\section{FINANCIAL SUPPORT AND SPONSORSHIP}

Nil

\section{CONFLICTS OF INTEREST}

There are no conflicts of interest.

\section{REFERENCES}

1. Resolution and Regulation of Inflammation BD Levy and CN Serhan, Harvard Medical School, Boston, MA, Pathobiology of Human Disease: A Dynamic Encyclopedia of Disease Mechanisms http://dx.doi.org/10.1016/B978-0-12-3864567.01811-6

2. Hamberg M, Samuelsson B. Prostaglandin endoperoxides. Novel transformations of arachidonic acid in human platelets. Proc Natl Acad Sci U S A. 1974;71(9):3400-3404.

3. Fierro IM, Serhan CN. Mechanisms in antiinflammation and resolution: the role of lipoxins and aspirin-triggered lipoxins. Braz J Med Biol Res. 2001;34(5):555-566.

4. Serhan CN, Hamberg M, Samuelsson B. Lipoxins: novel series of biologically active compounds formed from arachidonic acid in human leukocytes. Proc Natl Acad Sci U S A. 1984;81(17):5335-5339.

5. Serhan CN, Savill J. Resolution of inflammation: the beginning programs the end. Nat Immunol. 2005;6(12):1191-1197.

6. Kantarci A, Van Dyke TE. Lipoxins in chronic inflammation. Crit Rev Oral Biol Med. 2003;14(1):4-12.

7. Chandrasekharan J, Sharma-Walia N. Lipoxins: nature's way to resolve inflammation. J Inflamm Res. 2015;8:181-192 
Jaishree et al: Lipoxins for Resolution of Inflammation

8. Resolution and Regulation of Inflammation BD Levy and CN Serhan, Harvard Medical School, Boston, MA, USA.

9. Schwab JM, Serhan CN. Lipoxins and new lipid mediators in the resolution of inflammation. Curr Opin Pharmacol. 2006 Aug;6(4):414-20. doi: 10.1016/j.coph.2006.02.006. Epub 2006 Jun 5. PMID: 16750421.

10. Van Dyke TE. The management of inflammation in periodontal disease. J Periodontol. 2008;79(8 Suppl):1601-1608. doi:10.1902/jop.2008.080173

11. Stewart AG. Mediators and receptors in the resolution of inflammation: drug targeting $\begin{array}{lllll}\text { opportunities. } & \mathrm{Br} & \mathrm{J} & \text { Pharmacol. } 2009\end{array}$ Oct;158(4):933-5. doi: 10.1111/j.14765381.2009.00484.x. PMID: 19845683; PMCID: PMC2785516.

12. Chen H, Takahara M, Xie L, Takeuchi S, Tu Y, Nakahara T, Uchi H, Moroi Y, Furue M. Lipoxin $\mathrm{A}(4)$, a potential anti-inflammatory drug targeting the skin. J Dermatol Sci. 2011 Apr;62(1):67-9. doi: 10.1016/j.jdermsci.2011.01.011. Epub 2011 Feb 21. PMID: 21334856.

13. Serhan CN, Sheppard KA. Lipoxin formation during human neutrophilplatelet interactions. Evidence for the transformation of leukotriene A4 by platelet 12-lipoxygenase in vitro. J Clin Invest. 1990;85(3): 772-780.

14. Therapeutic Potential of Lipoxin A4 in Chronic Inflammation: Focus on Cardiometabolic Disease Ting Fu, Muthukumar Mohan, Eoin P. Brennan, Owen L. Woodman, Catherine Godson, Phillip Kantharidis, Rebecca H. Ritchie, and Cheng Xue Qin ACS Pharmacology \& Translational Science 20203 (1),43-55. DOI: 10.1021/acsptsci.9b00097

15. Van Dyke TE, Serhan CN. Resolution of inflammation: A new paradigm for the pathogenesis of periodontal diseases. J Dent Res 2003;82:82-90. 